\title{
Efficient Broadcasting for Route Discovery in Mobile Ad-hoc Networks
}

\author{
Haitham Y. Adarbah, Shakeel Ahmad, Member, IEEE, Bassel Arafeh, Member, IEEE, and Alistair Duffy, Fellow, IEEE
}

\begin{abstract}
Broadcasting is used in on-demand routing protocols to discover routes in Mobile Ad-hoc Networks (MANETs). On-demand routing protocols, such as AODV (Adhoc On-demand Distance Vector) routing, commonly employ pure flooding based broadcasting. However, pure flooding generates excessively redundant routing traffic that may lead to broadcast storm problem (BSP) and deteriorate the performance of MANETs significantly. Probabilistic broadcasting schemes were proposed in the literature to address BSP. However, these schemes do not consider thermal noise and interference which exist in real life MANETs, and therefore, do not perform well in real life MANETs. This paper presents a novel Channel Adaptive Probabilistic Broadcast (CAPB) scheme to disseminate RREQ packets efficiently. The proposed CAPB scheme determines the probability of rebroadcasting RREQ packets on the fly according to the current SINR (Signal to Interference plus Noise Ratio) and node density in the neighborhood. The proposed scheme and two related state of the art (SoA) schemes from the literature ( [1] and [2]) are implemented in the standard AODV routing protocol to replace the pure flooding based broadcast. Simulation results show that the proposed scheme outperforms the standard AODV and the two competitors in terms of routing overhead, throughput and end-to-end delay significantly.
\end{abstract}

Keywords: MANETs; Route Discovery; Channel Adaptive Probabilistic Broadcast; Broadcast Storm Problem; Probabilistic scheme.

\section{INTRODUCTION}

The proliferation of handheld gadgets, laptops, and smartphone devices, that are developed based on the IEEE 802.11 standard of wireless protocol have made Mobile Adhoc Networks (MANETs) an active area of research over the past two decades. A MANET is a self-configuring, self-healing and infrastructure-less network of mobile nodes connected to each other over single-hop or multi-hop wireless links on adhoc basis [3] [4]. These characteristics of MANETs make them an ideal choice for a number of applications e.g., animal tracking, air surveillance using unmanned aerial vehicles (UAVs), communications in battlefields, rescue operation in disaster areas or quick deployment of networks without huge infrastructure cost.

MANET nodes can be located arbitrarily within an area and are free to move. The movement of MANET nodes changes the

Haitham Y. Adarbah, Shakeel Ahmad and Alistair Duffy are with the Faculty of Technology, De Montfort University, Leicester, UK

(sirhaytham@gmail.com; shakeel@dmu.ac.uk; apd@dmu.ac.uk).

Bassel Arafeh is with Department of Computer Science, Sultan Qaboos University, Muscat, Oman (arafeh@squ.edu.om) network topology dynamically. MANET nodes adapt to the changing topology by discovering new neighbors and establishing new routes to destination nodes [5]. A node may not communicate directly with a distant node due to limited transmission range, and may have to rely on other nodes to relay the message along the route to the final destination node. In this way, each node acts as a host node and a relay node to extend the reachability of other nodes.

When a node wants to send data to a remote node, first, it finds out a set of relay nodes between itself and the remote node. The process of finding the optimal set of relay nodes between the source node and the destination node is called route discovery. Node mobility, limited battery power and the error-prone nature of wireless links are the main challenges in designing an efficient routing protocol in MANETs.

A number of routing protocols have been proposed in the literature [6][7]. These protocols generally fall into three categories namely table-driven (proactive), on-demand (reactive) and hybrid routing protocols. Table-driven routing protocols aim to maintain routes to all possible destinations in the network at all times. Examples of table-driven routing protocols include OLSR (Optimized Link State Routing) [8] and DSDV (Destination-Sequenced Distance-Vector) routing [9]. In contrast to table-driven approach, on-demand routing protocols, e.g., AODV (Ad-hoc On-demand Distance Vector) routing [10], DSR (Dynamic Source Routing) [6], and ABR (Associativity-Based Routing) [11], discover a route only when it is needed. Hybrid routing protocols, e.g., ZRP (Zone Routing Protocol) [12] and CEDAR (Core-Extraction Distributed Ad-hoc Routing) [13] combine the features of both proactive and reactive routing protocols. Interested reader can find survey in this reference [14].

In on-demand routing protocols, the routing process consists of two phases namely route-discovery and routemaintenance. These protocols rely on broadcasting for route discovery. For example, in case of AODV routing protocol, a source node that needs to send data to a destination node triggers route discovery mechanism by broadcasting a special control packet, called Route Request (RREQ), to its neighbours who then rebroadcast the RREQ packet to their neighbours. The process continues until the RREQ packet arrives at the destination node. The destination node sends a control packet called Route Reply (RREP) that follows the path of RREQ in reverse direction and informs the source node that a route has been established. Since every node on receiving the RREQ for the first time rebroadcasts it, it 


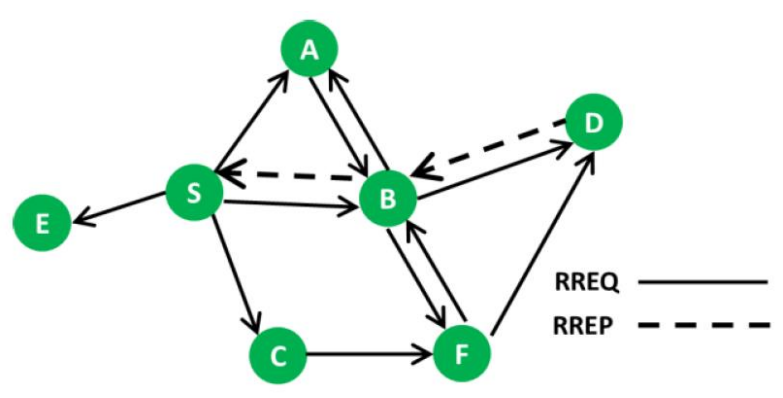

Fig.1: Route discovery mechanism in AODV routing protocol

requires T-2 rebroadcasts in a network of $\mathrm{T}$ nodes assuming the destination is reachable. This kind of broadcasting is called pure flooding and is depicted briefly in Figure 1 while details can be found in [10].

Pure flooding often results in substantial redundant transmissions because a node may receive the same packet from multiple other nodes. This phenomenon, commonly known as the broadcast storm problem (BSP) [1], triggers frequent contention and packet collisions leading to increased communication overhead and serious performance complications in densely populated networks. BSP equally affects the route maintenance phase during which routes are refreshed by triggering new route discovery requests to replace the broken routes.

A number of probabilistic broadcasting schemes have been proposed in the literature to address BSP. However, the performance of these schemes can be argued for real MANETs because these schemes either ignored thermal noise and interference at all [15] [16] or they used the noise drawn from a distribution rather than measuring it at lower layers [2]. Real life MANETs are noisy and the communication is not error free. A number of channel impairments like noise, cochannel interference, signal attenuation, fading and user mobility affect the transmission. This paper presents a novel Channel Adaptive Probabilistic Broadcasting (CAPB) scheme that adapts the probability of rebroadcasting RREQ packets dynamically according to the thermal noise, co-channel interference and node density in neighbourhood. The proposed scheme is implemented in the network simulator ns-2 and its performance has been compared with SoA schemes in terms of routing overhead, throughput and end-to-end delay. Simulation results showed that the proposed scheme outperforms the SoA broadcast schemes significantly. The proposed scheme is light and does not require any extra information to be exchanged among the neighbours.

The rest of the paper is organized as follows: Section 2 presents the related work, Section 3 presents the proposed efficient broadcast scheme, and Section 4 presents simulation results and analysis followed by conclusions in Section 5 .

\section{RELATED WORK}

To address the broadcast storm problem, a number of broadcasting schemes have been proposed in literature [1] [17] [18]. These schemes generally fall in two categories namely deterministic and probabilistic broadcasting. Deterministic schemes (e.g., MPR [17] and Self Pruning Scheme [18]) exploit network information to make more informed decisions. However, these schemes carry extra overhead to exchange location and neighborhood information among nodes. On the other hand, the probabilistic schemes e.g., Fixed Probabilistic [1], and Counter Based Scheme [15] take local decisions to broadcast or not to broadcast a message according to a predetermined probability.

A fixed probabilistic scheme is similar to simple flooding except that nodes rebroadcast with a predetermined probability. Cartigny and Simplot [16] presented an improved probabilistic/distance scheme combination where the retransmission probability value is calculated from the number of neighbors which are considering retransmission. This scheme was shown to achieve significant reduction in the number of retransmission packets. However, this scheme did not consider thermal noise and co-channel interference which cannot be ignored in real life MANETs.

Zhang and Agrawal [19] suggested a probabilistic scheme that dynamically modifies the rebroadcasting probability based on the node distribution and the node movement by considering local information but without needing any distance measurements or exact location determination devices. Their results showed an improvement in performance when compared to both pure flooding and static probabilistic schemes. However, the effects of noise and interference were ignored. The same authors (in another work [20]) suggested a levelled probabilistic routing scheme for MANETs. In this scheme, mobile hosts are divided into four groups and different rebroadcast probabilities are assigned to each group. The results showed gains in throughput.

Mohammed et al. [15] suggested a probabilistic counterbased scheme that reduces the retransmission of RREQ packets during the route discovery phase. The results revealed an enhancement in the performance of AODV in terms of routing overhead, MAC collisions, and end-to-end delay while still achieving a good throughput. However, this approach did not consider thermal noise plus interference.

Al-Bahadili and Sabri [2] proposed a probabilistic algorithm for route discovery based on the noise-level called dynamic noise-dependent probabilistic (DNDP). In their approach, they used the noise-level value drawn from a distribution rather than measuring it at lower layers. The simulation results showed that the suggested algorithm presented higher network reachability than the dynamic probabilistic algorithm with a reasonable increase in the number of retransmissions for a wide range of noise-levels.

Linfoot, et al.[21] studied the effect of physical and virtual carrier sensing on the AODV routing protocol and showed that the route discovery mechanism is affected by the interference when the number of nodes increases.

In wireless networks, physical layer characteristics affect the higher layer protocols. This shows a great potential of exploiting cross layer optimization approaches. Takai et al. [22] studied the role of physical layer modelling in evaluating the performance of higher layer protocols and showed that the physical layer modelling plays a key role even though the higher layer protocols do not interact with the physical layer directly. 


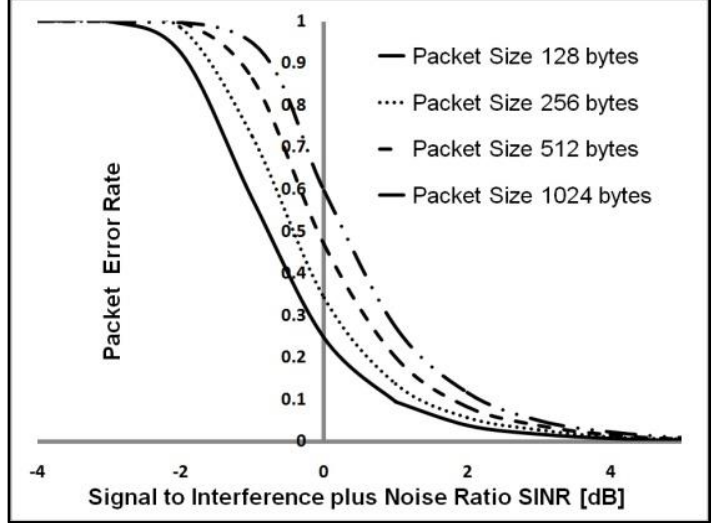

Fig.2: PER-SINR relationship for different packet sizes in 802.11g [23].

Alnajjar and Chen [24] stated a cross-layer mechanism wherein the routing protocols adapt to the current Signal to Noise Ratio (SNR). This approach was implemented in DSR protocol and was shown to enhance the performance.

To the best of the authors' knowledge, no previous work on probabilistic broadcast in route discovery mechanism has considered the effects of thermal noise, co-channel interference, and node density in the neighbourhood simultaneously to address the BSP.

\section{PROPOSED BROADCAST SCHEME}

The proposed Channel Adaptive Probabilistic Broadcast (CAPB) scheme adjusts the probability of rebroadcasting RREQ packets dynamically according to the SINR and node density in the neighborhood. These two factors affect the efficacy of disseminating RREQ packets significantly.

When a node receives a RREQ packet, it obtains the SINR value, as measured at the physical layer. Since, the Packet Error Rate (PER) is closely related to SINR and packet size [23], the scheme then infers the PER using the relationship shown in Figure 2. If the PER is higher, then the probability of receiving the same RREQ packet by the neighboring nodes is low. In this case, naturally the lucky node that has received the RREQ should rebroadcast the RREQ with high probability to increase the dissemination of this particular RREQ packet. On the other hand, a low PER implies that many nodes in the neighborhood have also received this RREQ packet with high probability, therefore the rebroadcast probability should be relatively low to avoid the BSP. Node density in the neighborhood is equally important. In a densely populated area, not all nodes need to rebroadcast to avoid redundancy and the risk of increased collision. On the other hand, in a sparsely populated area more nodes should rebroadcast the RREQ packet to ensure dissemination of the RREQ packet. Here we consider only the number of nodes in the transmission range of the node receiving the RREQ packet to determine the rebroadcast probability.

The proposed CAPB scheme has been shown in Figure 3. When node $R$ receives a RREQ packet, for which $R$ is not the destination node, it rebroadcasts the RREQ packet with probability $P_{r e b}$. To determine the value of $P_{r e b}$, node R determines the value of $N_{e f f}$ which is the number of effective nodes within its transmission range $r$ which have received the same RREQ packet. This is done as follows. Assume $N$ is the
Upon receiving a RREQ packet $m$ at a node $R$

Event: Node R receives RREQ packet $m$

if Node $\mathrm{R}$ is the destination node for RREQ $\mathrm{m}$ then Send RREP

else

Calculate $N_{b}$

Obtain SINR and infer PER

Calculate $N_{\text {eff }}$ using eq. 4

Calculate $P_{\text {reb }}$ from eq. 6

Generate a random number $\boldsymbol{\delta}$ between 0 and 1.0

if $\boldsymbol{\delta}<=P_{r e b}$ then

Broadcast the RREQ message $m$

else

Drop the RREQ message $m$

end if

end if

End if

Fig.3: Proposed CAPB scheme

total number of nodes within the transmission range of node R. We use Hello Packets to infer the value of $N$. The number of nodes $N_{b}$ which are located within the transmission range of both nodes $\mathrm{R}$ and node $\mathrm{S}$ can be calculated from the overlapped area A of the two circles as shown in Figure 4. Using geometry, the overlapped area A can be given by

$$
A=(\theta \times \pi / 180-\sin \theta) \times r^{2}
$$

Here $\theta$ is the angle of the circular segment in degrees. Note that $\theta=120^{\circ}$ when node $\mathrm{R}$ is at the edge of the transmission range of node $S$, and $\theta=180^{\circ}$ when node $S$ is extremely close to node R. Node R estimates its distance from node $\mathrm{S}$ from the signal strength of the received RREQ packet and calculates the value of $\theta$ using simple trigonometric relations. To keep our scheme simple, we assume that nodes are uniformly distributed. With this assumption, the value of $N_{b}$ can be given by

$$
N_{b}=N \times A / \pi r^{2}
$$

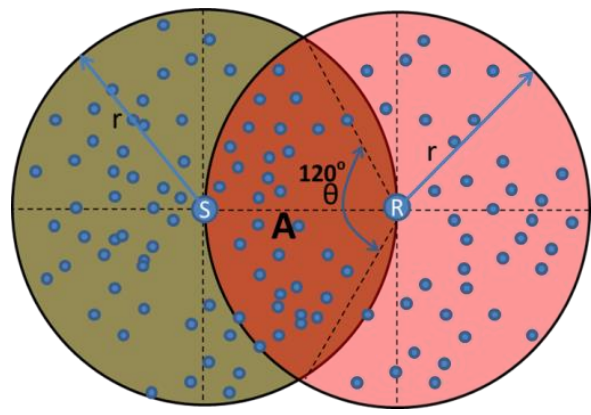

Fig.4: Node R receives RREQ from node S. 
To take into account the effects of thermal noise and cochannel interference, node $\mathrm{R}$ obtains the SINR from the physical layer at the time of receiving the RREQ packet and infers the PER using the relationship shown in Figure 2. The value of $N_{e f f}$ is then given by

$$
N_{e f f}=N_{b} \times(1-P E R)
$$

Equation (3) can be simplified to

$$
N_{e f f}=N \times\left(\frac{\theta}{180}-\frac{\sin \theta}{\pi}\right)(1-P E R)
$$

A higher value of $N_{e f f}$ implies that more nodes have received the RREQ and consequently the value of $P_{r e b}$ should be lower and vice versa. This suggests an inverse relationship between $P_{r e b}$ and $N_{e f f}$.

$$
P_{r e b}=d \times \frac{1}{N_{e f f}}
$$

Here $d$ is a constant value representing the dissemination factor. The value of $d$ is greater than unity to compensate the PER. For very low $\left(\leq N_{l}\right)$ and very high $\left(\geq N_{u}\right)$ values of $N_{\text {eff }}$ equation (2) may not hold true so fixed values of $P_{\text {reb }}$ are used in those cases. In general $P_{r e b}$ can be given as follows:

$$
P_{\text {reb }}=\left\{\begin{array}{lr}
P_{\text {max }}, & \text { for } N_{\text {eff }} \leq N_{l} \\
d \times \frac{1}{N_{e f f}}, & \text { for } N_{l}<N_{e f f}<N_{u} \\
P_{\text {min }}, & \text { for } N_{\text {eff }} \geq N_{u}
\end{array}\right.
$$

Appropriate values of $N_{l}, N_{u}$ can be derived from an estimated maximum and minimum possible node density and the transmission range of nodes. The implementation of the proposed scheme and its performance evaluation is presented in the next section.

\section{PERFORMANCE EVALUATION OF THE CAPB ALGORITHM}

The proposed CAPB scheme has been evaluated and compared with three related broadcasting schemes. The first one is pure flooding that is part of the standard AODV routing protocol. The second one is the fixed probabilistic scheme [1] denoted by AODV-P where $\mathrm{P}$ shows the rebroadcast probability. The third scheme is DNDP (Dynamic NoiseDependent Probabilistic) scheme of [2].

\section{A. Simulation Setup}

We used ns-2 simulator (2.35v) to implement and evaluate the proposed scheme in MANETs using AODV routing protocol. Standard AODV uses pure flooding. The proposed CAPB scheme and the two other schemes from the literature (AODV-P and AODV-DNDP) have been implemented in the route discovery process of AODV. In AODV-P scheme, the value of $\mathrm{P}$ is set to 0.6 after running extensive simulation with a range of values for $\mathrm{P}$ and choosing the one giving the best performance. The parameters of AODV-DNDP scheme follow recommendations in [2]. For CAPB, we set $N_{l}=7, N_{u}=$ $16, P_{\max }=0.7, P_{\min }=0.3$ and $d=5$. These values are partly heuristic and partly simulation guided.

The MANET related simulation parameters generally follow [2][25][21]. The radio propagation is based on 2-ray Ground Reflected Model. The network bandwidth is set to 6 Mbps and the medium access control (MAC) protocol is simulated using the ns2 library dei80211mr [23]. This library calculates the PER using pre-determined curves (PER Vs. SINR) for the given packet size (shown in Figure 2). The SINR value is computed from the received signal strength, thermal noise and co-channel interference. Thermal noise is set to $-95 \mathrm{dBm}$ following recommendations in [26].

The node mobility is modeled using Random Waypoint [27] mobility model with variable node speed and pause time set to zero. Nodes are placed randomly in an area of $1000 \times 1000$ square meters. Transmission power, path loss and receive power are set such that the effective transmission range is $250 \mathrm{~m}$. Each node has a FTP (File Transfer Protocol) agent attached to it such that node $\mathrm{i}$ is downloading a file of infinite size from node $i+M / 2$ for $i=1,2, \ldots, M / 2$ where $M$ is the total number of nodes.

\section{B. Simulation results and analysis}

We used two sets of simulations, the density-scenario and the mobility-scenario. The density-scenario uses a fixed node speed of $5 \mathrm{~m} / \mathrm{s}$ for each node and the number of nodes is varied. The mobility-scenario uses fixed number of nodes (set to 100) and node speed is varied. Simulation results are obtained by averaging the results of 30 runs, each using a different seed value and lasting for 800 seconds. The seed value is used in the mobility model to yield different mobility profiles and to set the initial location for each node.

\section{1) Routing Overhead}

Routing overhead is defined as the ratio of the number of routing packets (control packets) transmitted per data packet received. Figure 5 and Figure 6 show the average routing overhead as a function of node density and node speed respectively. In general the average routing overhead increases with increasing node density because higher number of neighboring nodes lead to higher contention and PER which results in redundant retransmission of control packets. Similarly, increasing node speed makes the network topology more dynamic. Routes get expired quickly and new route discovery mechanism is triggered more frequently to replace the expired routes.

Increasing the number of RREQ broadcasts increases the reachability of nodes on one hand but on other hand, it may increase the co-channel interference leading to higher PER which may limit the reachability and require restart the route discovery process. This is the reason of higher overhead of pure AODV scheme. 


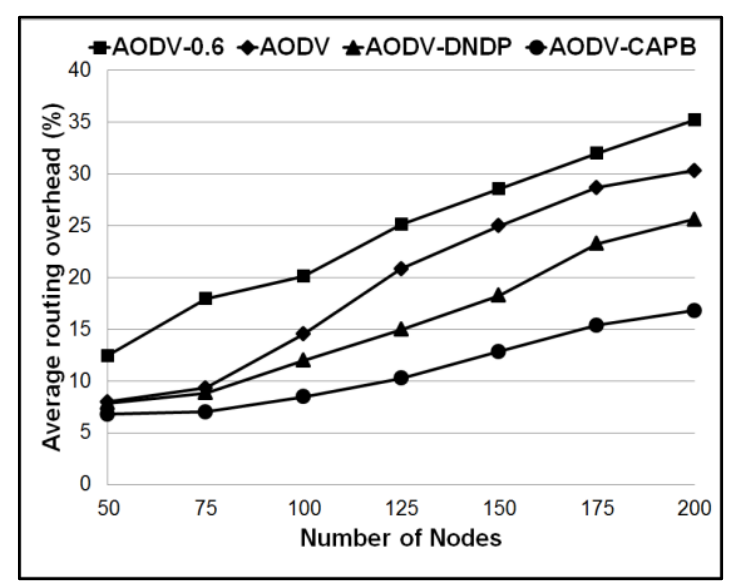

Fig.5: Routing overhead for different number of nodes

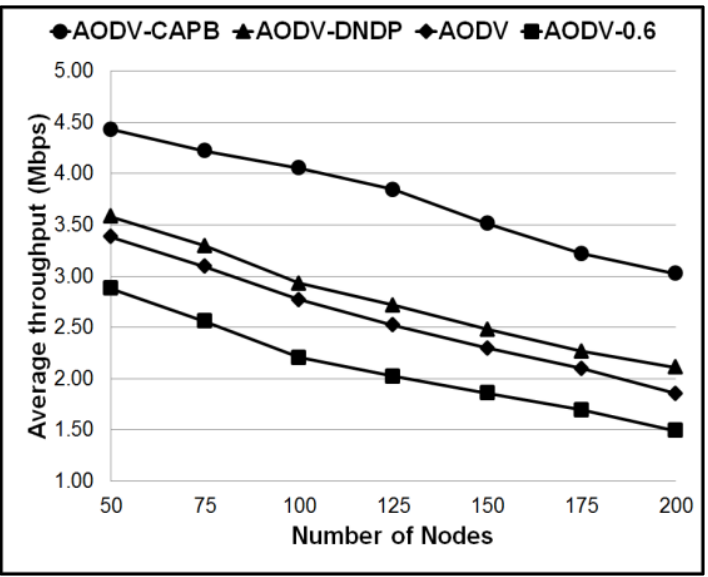

Fig.7: Average throughput for different number of nodes

Fixed probabilistic scheme (AODV-0.6) limits the number of RREQ blindly which often limits the reachability of RREQ packets to the destination node and route discovery mechanism has to be triggered more frequently leading to higher overhead. It is interesting to note that the routing overhead of pure AODV is lower than AODV-0.6 scheme. In fact, thermal noise plus co-channel interference act as natural limiters for the traffic; the former is static while the latter is adaptive because it increases with traffic intensity. This reduces the chances of getting duplicate RREQs from the neighboring nodes and adapts to the traffic intensity. In presence of natural and adaptive limiters (thermal noise and co-channel interference), the artificial limiter (reducing the rebroadcast probability without considering the effect of interference and thermal noise), does not work well because it limits the reachability of RREQs independent of the traffic intensity. Nodes have to try several times before they get a valid route which increases the routing overhead.

In AODV-DNDP, the probability is not fixed and is drawn from a distribution without considering the current level of noise and interference. The proposed CAPB scheme is able to achieve at least 33\% lower overhead as compared to the best competitor scheme almost across the whole range on $\mathrm{x}$-axis in Figure 5 and 6 . The savings in routing overhead increases with the increase in node density and node speed.

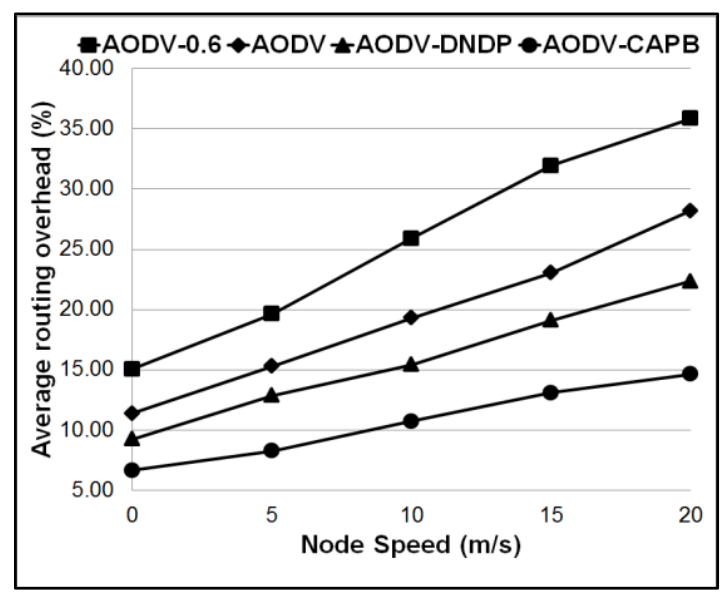

Fig.6: Routing overhead for different values of node speed

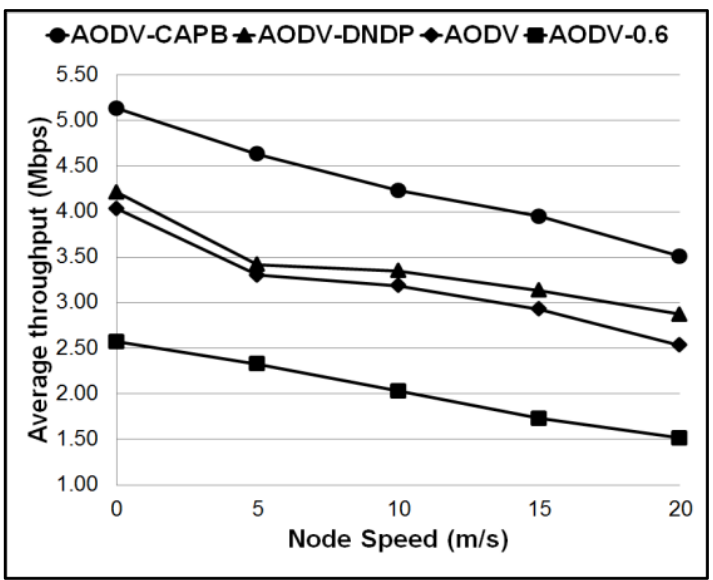

Fig.8: Average throughput for different values of node speed

\section{2) Throughput}

Throughput is defined as the amount of data received by a node per unit time. Figure 7 shows the average throughput, measured at the application layer, for all nodes as a function of number of nodes, and Figure 8 shows the average throughput as a function of node speed.

In general, the average throughput decreases by increasing the number of nodes due to increased contention ratio and higher collision rate. The average throughput also decreases with increasing node speed because routes are broken more frequently due to changing neighborhood and network topology causing a temporary pause in data transmission till the new route is established. The time required to establish new routes to replace the broken ones and the routing overhead affect the throughput significantly. Inefficient or blind decision of rebroadcasting the RREQ packets may not result in a successful route establishment at first attempt and the process may have to be initiated repeatedly. This would increase the time to establish a route from the source node to the destination node. The FTP application has to wait longer before it could start sending data. Moreover, node mobility invalidates old routes more frequently and interrupts the data supply until an alternative route is established. The proposed algorithm is able to achieve throughput gain of at least $30 \%$ over the other schemes. This is because the rebroadcasting decision in CAPB takes into account SINR and node density 


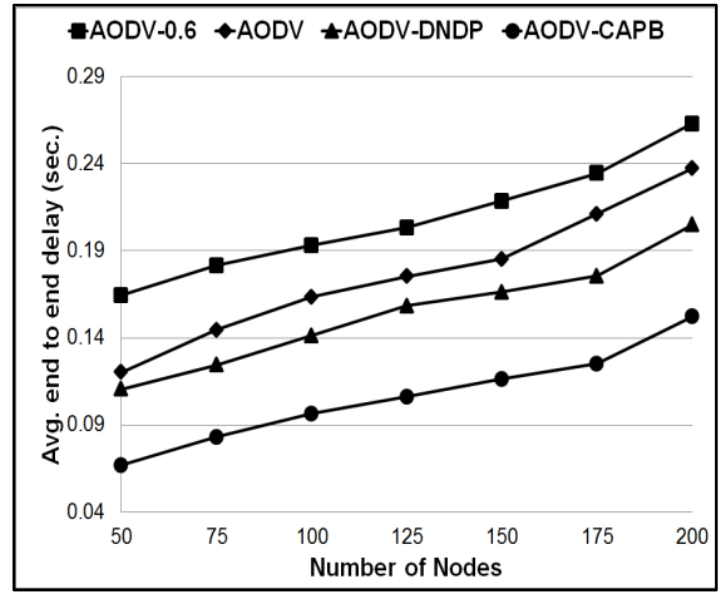

Fig.9: Average end-to-end delay for different number of nodes

in the neighborhood which increases the reachability of RREQ to the destination node while keeping the routing overhead at minimum.

\section{3) Average End-to-End Delay}

The average end-to-end delay shows the time a packet takes to reach from the source node. It includes all possible delays caused by buffering during route discovery, queuing at the interface queue, retransmission delays at the MAC, propagation delay and transmission delay.

Figure 9 shows the end-to-end delay for data packets averaged over all nodes as a function of number of nodes and Figure 10 presents the same as a function of node speed. It can be seen that for all schemes, the average end-to-end delay increases with increasing number of nodes and node speed.

By increasing the number of nodes, contention increases leading to higher queuing delay at the transmitter's buffer and higher packet loss rate due to increased number of collisions. A data packet may need to be retransmitted multiple times. With increased mobility, route breaking and repairing takes places more often leading to higher average delay. The proposed CAPB scheme outperforms the other schemes by at least $40 \%$ lower average end-to-end delay. It is possible because the proposed scheme produces lower routing traffic which helps to decrease the contention and collision, and it increases the reachability of RREQ packets to the destination which helps to establish or repair routes faster.

\section{CONCLUSION AND FUTURE WORK}

Broadcasting is used in the route discovery phase of ondemand routing protocols in MANETs. Many on-demand routing protocols e.g., AODV use pure flooding to broadcast the RREQ packet. However, pure flooding generates excessive control traffic which may lead to broadcast storm problem. A number of probabilistic broadcasting schemes have been proposed to limit the broadcast traffic but these schemes do not consider the thermal noise and the co-channel interference and hence do not perform well in realistic noisy MANETs. Node density in the neighbourhood is another important factor to determine the rebroadcast probability. This paper has presented a Channel Adaptive Probabilistic Broadcast (CAPB) scheme that adapts the rebroadcast probability to the thermal noise, co-channel interference and node density in the

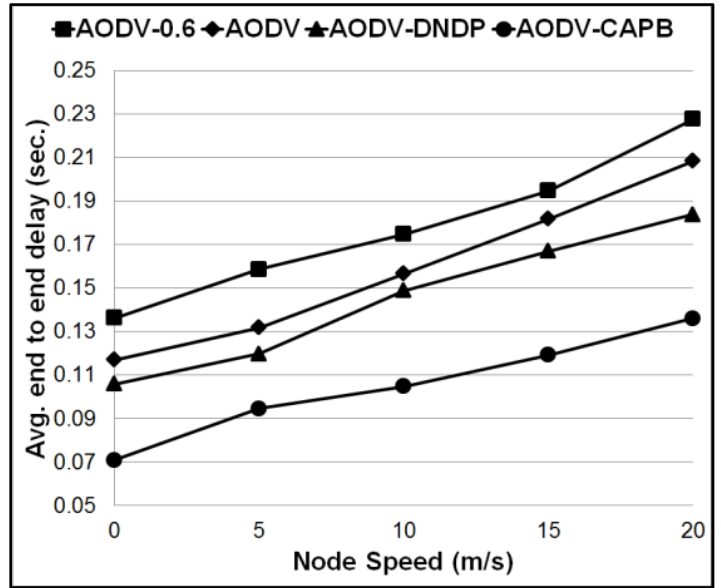

Fig.10: Average end-to-end delay for different values of node speed

neighbourhood dynamically. Simulations results have shown that the proposed CAPB scheme outperforms the standard AODV and the two related schemes significantly in terms of routing overhead, throughput, and end-to-end delay. The proposed scheme is simple and does not require any extra information to be exchanged among the neighbouring nodes.

Potential future research directions to extend this work include designing a systematic approach to adjust the minimum and maximum values of rebroadcast probability with the node density adaptively, and to testify the proposed scheme using other on-demand routing protocols e.g., DSR.

\section{REFERENCES}

[1] S. Ni, Y. Tseng, and Y. Chen, "The broadcast storm problem in a mobile ad hoc network," Wirel. Networks, no. 8, pp. 153-167, 2002.

[2] H. Al-Bahadili and A. Sabri, "A Novel Dynamic Noise-Dependent Probabilistic Algorithm for Route Discovery in MANETs," Int. J. Bus. Data Commun. Netw., vol. 7, no. 1, pp. 52-67, 2011.

[3] J. Liu, X. Jiang, and S. Member, "Throughput Capacity of MANETs with Power Control and Packet Redundancy," IEEE Trans. Wirel. Commun., vol. 12, no. 6, pp. 3035-3047, 2013.

[4] IETF, "Mobile ad-hoc networks (MANETs)," 2015. [Online]. Available: http://www.ietf.org/proceedings/53/179.htm. [Accessed: 30-Mar-2015].

[5] C. Ni, H. Liu, A. G. Bourgeois, and Y. Pan, "An enhanced approach to determine connected dominating sets for routing in mobile ad hoc networks," Int. J. Mob. Commun., vol. 3, no. 3, pp. 287 - 302, 2005.

[6] N. Sarkar and W. Lol, "A study of manet routing protocols: Joint node density, packet length and mobility," in IEEE Symposium on Computers and Communications (ISCC), 2010, pp. 515-520.

[7] M. Abolhasan, "A review of routing protocols for mobile ad hoc networks," Ad Hoc Networks, vol. 2, no. 1, pp. 1-22, Jan. 2004.

[8] T. Clausen and P. Jacquet, "Optimized Link State Routing Protocol (OLSR)," RFC 3626, IETF Network Working Group, 2003. [Online]. Available: http://www.ietf.org/rfc/rfc3626.txt. [Accessed: 23-Feb-2015].

[9] C. E. Perkins and P. Bhagwat, "Highly Dynamic ( DSDV ) for Mobile Computers Routing," in In Proceedings of the SIGCOMM '94 Conference on Communications Architectures, Protocols and Applications, 1994, pp. 234-244

[10] S.-J. Lee, E. M. Belding-Royer, and C. E. Perkins, "Ad hoc ondemand distance-vector routing scalability," ACM SIGMOBILE Mob. Comput. Commun. Rev., vol. 6, no. 3, p. 94, Jun. 2002.

[11] C. K. Toh, "Associativity-Based Routing for Ad Hoc Mobile Networks," Wirel. Pers. Commun., vol. 4, no. 2, pp. 1-36, 1997.

[12] Z. J. Haas and M. R. Pearlman, "The performance of query control schemes for the zone routing protocol," in Proc. ACM SIGCOMM '98, 1998, pp. 167-177. 
[13] P. Sinha, R. Sivakumar, and V. Bahrghavan, "CEDAR: A Core Extraction Distributed Ad Hoc Roouting Algorithm," IEEE J. Sel. Areas Commun., vol. 17, no. 8, pp. 1454-1466, 1999.

[14] A. Boukerche, B. Turgut, N. Aydin, M. Z. Ahmad, L. Bölöni, and D. Turgut, "Routing protocols in ad hoc networks: A survey," Comput. Networks, vol. 55, no. 13, pp. 3032-3080, 2011.

[15] A. Mohammed, M. Ould-Khaoua, L. M. Mackenzie, C. Perkins, and J.-D. Abdulai, "Probabilistic counter-based route discovery for mobile ad hoc networks," in Proceedings of the 2009 International Conference on Wireless Communications and Mobile Computing Connecting the World Wirelessly - IWCMC '09, 2009, pp. 13351339.

[16] J. Cartigny and D. Simplot, "Border Node Retransmission Based Probabilistic Broadcast Protocols in Ad-Hoc Networks," in The 36th Annual Hawaii International Conference on System Sciences (HICSS'03), 2002, pp. 6-9.

[17] A. Qayyum, L. Viennot, and A. Laouiti, "Multipoint relaying for flooding broadcast messages in mobile wireless networks," in Proceedings of the 35th Annual Hawaii International Conference on System Sciences, 2002, pp. 3866-3875.

[18] H. Lim and C. Kim, "Multicast tree construction and flooding in wireless ad hoc networks," in Proceedings of the ACM International Workshop on Modeling, Analysis and Simulation of Wireless and Mobile Systems (MSWIM), 2000.

[19] Q. Zhang and D. P. Agrawal, "Dynamic probabilistic broadcasting in MANETs," J. Parallel Distrib. Comput., vol. 65, no. 2, pp. 220 233, Feb. 2005.

[20] Q. Zhang and D. P. Agrawal, "Analysis of Leveled Probabilistic Routing in Mobile," in IEEE International Conference on Communications, 2004, pp. 3896-3900.

[21] S. Linfoot, H. Y. Adarbah, B. Arafeh, and A. Duffy, "Impact of Physical and Virtual Carrier Sensing on the Route Discovery Mechanism in Noisy MANETs," IEEE Trans. Consum. Electron., vol. 59, no. 3, pp. 515-520, 2013.

[22] M. Takai, J. Martin, and R. Bagrodia, "Effects of wireless physical layer modeling in mobile ad hoc networks," in Proceedings of the 2nd ACM international symposium on Mobile ad hoc networking \& computing, 2001, pp. 87-94.

[23] DEI, "NS2 Library: dei80211mr," 2014. [Online]. Available: http://www.isi.edu/nsnam/ns/doc/node193.html. [Accessed: 17-Feb2015].

[24] F. Alnajjar and Y. Chen, "SNR/RP Aware Routing Algorithm: Cross-Layer Design for MANETs," Int. J. Wirel. Mob. Netowrks, vol. 1, no. 2, pp. 127-136, 2009.

[25] Y. Tseng, S. Ni, and E. Shih, "Adaptive Approaches to Relieving Broadcast Storms in a Wireless Multihop Mobile," IEEE Trans. Comput., vol. 52, no. 5, pp. 545-557, 2003.

[26] X. S. Rajendra and V. Boppana, "On the impact of noise on mobile ad hoc networks," in Proceedings of the 2007 international conference on Wireless communications and mobile computing (IWCMC '07)., 2007, pp. 208-213.

[27] G. Lin, G. Noubir, and R. Rajamaran, "Mobility models for ad hoc network simulation," in Proceedings of 23rd conference of the IEEE communications society (INFOCOM 2004), 2004, pp. 454-463. 